\title{
Conservative treatment of coexisting microinvasive squamous and adenocarcinoma of the cervix: report of two cases and literature review
}

This article was published in the following Dove Press journal:

OncoTargets and Therapy

27 January 2016

Number of times this article has been viewed

\author{
Francesco \\ Sopracordevole ${ }^{1, *}$ \\ Jacopo Di Giuseppe ${ }^{2, *}$ \\ Silvia Cervo ${ }^{3,4}$ \\ Monica Buttignol' \\ Giorgio Giorda' \\ Andrea Ciavattini ${ }^{2}$ \\ Vincenzo Canzonieri ${ }^{3,5}$ \\ 'Gynecologic Oncology Unit, \\ Department of Surgical Oncology, \\ CRO Aviano National Cancer \\ Institute, Aviano, Pordenone, \\ 'Woman's Health Sciences \\ Department, Gynaecologic Section, \\ Polytechnic University of Marche, \\ Ancona, ${ }^{3} \mathrm{CRO}$-Biobank, ${ }^{4} \mathrm{Clinical}$ \\ Cancer Pathology, ${ }^{5}$ Pathology Unit, \\ CRO Aviano National Cancer \\ Institute, Aviano, Pordenone, Italy \\ *These authors contributed equally \\ to this work
}

\begin{abstract}
Coexistence of microinvasive squamous cell carcinoma (MISCC) and microinvasive adenocarcinoma (MIAC) of the cervix is a rare phenomenon with very few clinically significant cases described in the literature. While a conservative approach has been studied, and may be effective in MISCC, a lower number of studies that recommend conservative treatment are available for MIAC. We report two cases of synchronous cervix lesions in two separate foci, MISCC and MIAC, who underwent fertility-sparing treatment with long-term follow-up. We describe clinical, histological, and immunohistochemical features of the two cases. The first case is a 41-year-old female with a diagnosis of MIAC of endocervical type, grade 1 differentiation, with a stromal invasion, associated with a separate area of squamous cell carcinoma (International Federation of Gynecology and Obstetrics/TNM stage: pT1a1G1). The second case is a 45-year-old female with a diagnosis of plurifocal MISCC, associated with an MIAC of endocervical type with a stromal invasion (International Federation of Gynecology and Obstetrics/TNM stage: pT1a1G1). After multidisciplinary counseling, both patients accepted conization as definitive treatment. Eleven years after the conization, all tests (Papanicolaou smear, colposcopy, cervical curettage, and hybrid capture 2-human papillomavirus test) planned quarterly in the first year and every 6 months in the subsequent years were negative in both patients. In women affected by stage IA1 squamous cervical cancer coexisting with stage IA1 adenocarcinoma endocervical type, with clear margins, and without lymphovascular space invasion, cervical conization may be considered a fertility-preserving, safe, and definitive therapeutic option.
\end{abstract}

Keywords: cervical conization, cervical lesions, FIGO stage, microinvasive adenocarcinoma, microinvasive squamous cell carcinoma, uterine cervical neoplasms

\section{Background}

In the last few decades, the incidence of microinvasive cervical cancer (MICC), International Federation of Gynecology and Obstetrics (FIGO) stage pT1A1 and pT1A $2,{ }^{1}$ has increased significantly in developed countries. ${ }^{2}$

The large spreading of cytological-based screening programs led to an increase in the diagnosis of precancerous and microinvasive cervical lesions compared with frankly invasive tumors. Moreover, an increased number of microcarcinomas are diagnosed in young women in childbearing age, which coincides with the most common period for the onset of preneoplastic cervical lesions. ${ }^{3}$

Despite some controversies, mainly concerning patient selection criteria, the current trend is to treat the patients affected by MICC with conservative, less radical, fertility-sparing approaches. For patients with stage IA1 microinvasive squamous cell carcinoma (MISCC), the treatment may consist of cone biopsy with clear margins; 
this approach appeared to be safe and is reported in many studies in the literature..$^{4-7}$

More recently, a conservative approach was also proposed for patients who underwent accurate surgical staging for IA2 microinvasive carcinoma, with or without associated pelvic lymphadenectomy, which is mandatory in case of neoplastic lymph vascular space invasion (LVSI). Some authors described a conservative approach in IA2 microinvasive carcinoma considering the low incidence of both lymph node metastases and parametrial involvement. ${ }^{4,8-10}$

Glandular cervical lesions are more difficult to be diagnosed and are relatively infrequent, although recent reports indicate an increase of these diagnoses approaching approximately $27 \%$ of all cervical cancers. ${ }^{11}$ In particular, the frequency of microinvasive adenocarcinoma (MIAC) of the cervix is approximately $12 \%$ of all MICCs. ${ }^{12}$

However, less is known about MIAC compared with their squamous counterpart probably due to MIAC cellular heterogeneity, supported by different histological glandular types, different natural history, and different oncogenetic pathways. MIAC is not always linked to human papillomavirus (HPV) infection and rarely arises in glandular crypts distant from the squamocolumnar junction.

Microscopically, the endocervical "usual type" is the most common histotype, rising close to the squamouscolumnar junction (SCJ) in more than $90 \%$ of cases, and is usually associated with better prognosis. Conversely, other histotypes, such as endometrioid or clear cell adenocarcinomas, may arise in any place along the cervical canal and represent tumor types usually showing early recurrence and worse survival in stage IA1 and IA2. ${ }^{13}$ Interestingly, MIAC is occasionally found in cone biopsy performed for different pathological conditions, such as adenocarcinoma in situ (AIS), glandular cervical dysplasia, and squamous intraepithelial lesions (SILs). In this last situation, the SIL has been considered a "marker lesion" for the presence of a hidden glandular lesion, with which it may occasionally share the expression of the same HPV genotype. In these cases, the squamous lesions may produce high viral load and shedding of thousands of HPV particles at the SCJ that may lead to infection of adjacent glandular epithelium or undifferentiated stem cells. ${ }^{14}$ Accordingly, cancer is thought to arise from the transformation zone between glandular and squamous epithelia, where stem-like cells are thought to reside, and which is the most common site of HPV infection resulting in the onset of one of the two types of cancer. ${ }^{15}$ Therefore, coexistence of MISCC and MIAC is a biological unique situation in need of investigation.
In our institution, from January 1, 1995 to December 31, 2014, 1,314 women underwent laser cone biopsy for highgrade squamous intraepithelial lesion (H-SIL)/cervical intraepithelial neoplasia 2 or 3 (CIN 2 or 3), or because of suspected AIS or persistent low-grade SIL after unsatisfactory colposcopy.

Among these patients, 91 invasive squamous cell carcinomas, 29 invasive adenocarcinomas, two endometrioid carcinomas, one serous carcinoma, and four adenosquamous carcinomas were found.

We report here the pathological characterization of two cases of synchronous lesions in two separate foci (MISCC and MIAC), in women who underwent fertility-sparing treatment with clinical long-term follow-up.

Written informed consent was obtained from the patients for publication of these case reports and any accompanying images. Approval from an ethics committee was not required due to being a retrospective study and the two cases are anonymous.

\section{Case presentations Case I}

A 41-year-old female, para 2, underwent a Papanicolaou (Pap) smear 36 months after her last negative test. The diagnosis was atypical glandular cells, favor neoplasia. She did not smoke, and the HIV test was negative. She had taken contraceptive pills for 2 years, until the age of 38 . A colposcopy was performed 15 days after diagnosis; it was not satisfactory, with SCJ not fully visible, and showing grade 1 abnormal transformation zone because of the extension of thin acetowhite iodine-negative epithelium toward the cervical canal. No atypical vessels were found inside the glandular epithelium.

Colposcopic direct biopsy was performed and a diagnosis of suspected endocervical glandular dysplasia was rendered. Endocervical and endometrial curettages were negative. The patient also underwent hysteroscopy showing normal findings.

In accordance with the guidelines of the European Society for Colposcopy and Cervical Pathology, ${ }^{16}$ a diagnostic cone biopsy was performed to define the lesion and to exclude invasive diseases.

The colposcopic guide laser conization was performed with a Surgilase $40 \mathrm{CO}_{2}$ laser, at a power setting of $40 \mathrm{~W} / \mathrm{cm}^{2}$, connected to a micromanipulator mounted on a Zeiss colposcope with a focal spot size of $0.2 \mathrm{~mm}$, under local anesthesia (cervical injections of $3.0-5.0 \mathrm{~mL}$ of a $2 \%$ lidocaine). There were no intraoperative or postoperative complications, and there was no need for stitches. Blood loss was negligible. The specimen of conization was a truncated cone, $2 \mathrm{~cm}$ in 
height, with a $2 \mathrm{~cm}$ larger base and a $0.7 \mathrm{~cm}$ shorter base, weighing $6 \mathrm{~g}$. For the histological analysis, longitudinal sections were taken at regular $1.5 \mathrm{~mm}$ intervals across the specimen. Sections of $5 \mu \mathrm{m}$ thickness were cut from the formalin-fixed paraffin-embedded tissue blocks and stained with hematoxylin and eosin (Figure 1).

The pathologic diagnosis was MIAC of endocervical type, grade 1 differentiation, with a stromal invasion of 0.4 $\mathrm{mm}$ in depth, and a $4 \mathrm{~mm}$ largest superficial extension, close to the SCJ, without LVSI, associated with a separate area of MISCC (3.0 mm largest superficial extension and $1.0 \mathrm{~mm}$ in depth). Immunohistochemistry showed positivity for $\mathrm{p} 16$ (Figures 2 and 3) in both squamous and glandular components. High molecular cytokeratin $(34 \beta E 12)$ was positive in the squamous component, and in situ hybridization was performed without a conclusive result because of the presence of dot-like purple precipitate in squamous, glandular, as well as stromal cell nuclei indicating nonspecific hybridization.

The section margins of the cone were clear. Definitive FIGO/TNM stage was pT1a1G1 (TNM 7th edition). After multidisciplinary counseling, the patient accepted conization as definitive treatment. She also declared to be available for follow-up with Pap smear, colposcopy, cervical curettage, and after a year hybrid capture 2-HPV test. Tests were performed quarterly in the first year and then every 6 months in the subsequent years. HPV testing became negative after 1 year of conization. Eleven years after the conization, all tests (Pap smear, colposcopy, and cervical curettage) were negative.

\section{Case 2}

A 45-year-old female, nulliparous, underwent a Pap smear 36 months after her last negative test. The diagnosis was

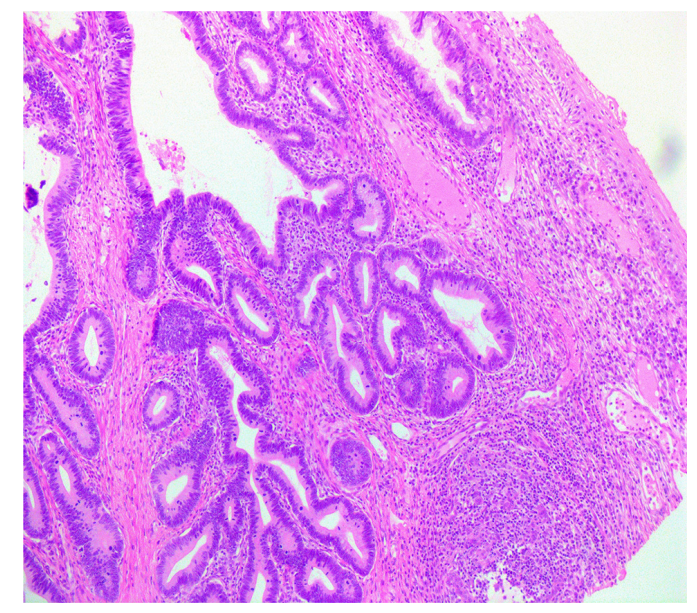

Figure I Well-differentiated adenocarcinoma infiltrating cervical stroma and associated microinvasive squamous cell carcinoma (case I, original magnification $\times 10$ ).

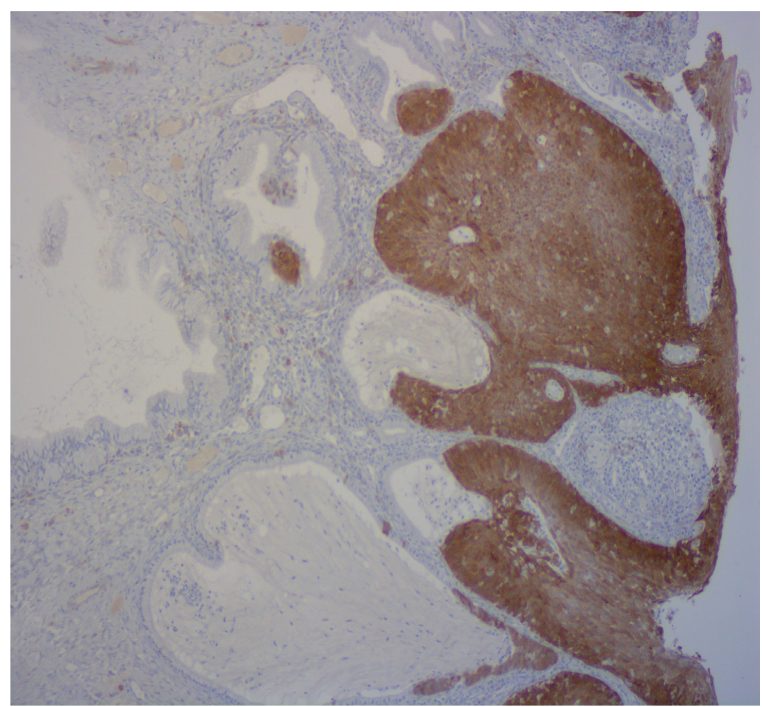

Figure 2 pl6 immunopositive staining in squamous cell carcinoma (case I, original magnification $\times 10$ ).

H-SIL. She reported smoking about five cigarettes daily. HIV test was negative. She never took hormone therapy. A colposcopy was performed 20 days later; it was satisfactory, with SCJ fully visible, and showed grade 2 abnormal transformation zone because of the presence of irregular punctuation, irregular mosaic, and atypical vessels. Colposcopic direct biopsy allowed a pathological diagnosis of suspected H-SIL. Therefore, a diagnostic cone biopsy was indicated to define the lesion and to exclude invasive disease. The procedure was the same as in case 1 and no intraoperative or postoperative complications were recorded.

The specimen of conization was a truncated cone, $0.7 \mathrm{~cm}$ in height, with a $3 \mathrm{~cm}$ larger base and a $0.7 \mathrm{~cm}$ shorter base, weighing $6 \mathrm{~g}$. For the histological analysis, longitudinal

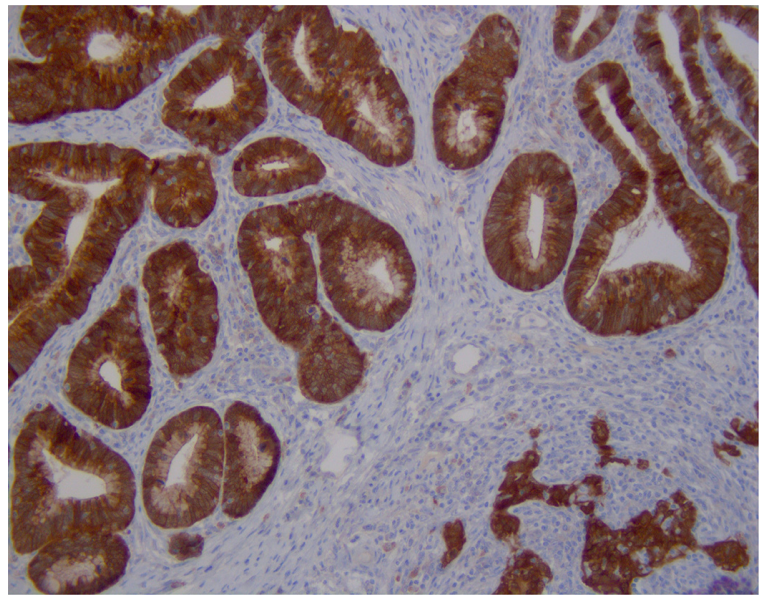

Figure 3 pl6 immunopositive staining in adenocarcinoma cells (case I, original magnification $\times 10$ ). 
sections were taken at regular $1.6 \mathrm{~mm}$ intervals across the conization and the whole specimen was included. The submitted tissue was processed, with the same method as the previous case, and stained with hematoxylin and eosin (Figure 4). The pathologic diagnosis was a plurifocal MISCC extended $0.3 \mathrm{~mm}$ in depth and $2 \mathrm{~mm}$ in width. It was associated with an MIAC of endocervical type with a stromal invasion of $1 \mathrm{~mm}$ in depth, and a $2 \mathrm{~mm}$ largest superficial extension, close to the SCJ, without LVSI. Immunohistochemistry showed positivity for p16 (Figure 5) and 34ßE12 (Figure 6) in both squamous and glandular components. In situ hybridization showed inconclusive results, as in the previous case (case 1). The section margins of the cone were clear. Definitive FIGO/TNM stage was pT1a1G1 (TNM 7th edition). After multidisciplinary counseling, the patient accepted conization as definitive treatment. She also declared to be available for follow-up with Pap smear, colposcopy, cervical curettage, and after a year, hybrid capture 2-HPV test (the latter showed a negative result after 1 year from the conization). Tests were performed quarterly in the first year and then every 6 months in the subsequent years. Eleven years after the conization, all tests (Pap smear, colposcopy, and cervical curettage) were negative.

\section{Discussion}

A double primary carcinoma in one organ is defined as a condition when the two carcinomas are histologically distinct and separated from each other by stroma or basal lamina. ${ }^{17}$

In our two cases, we found two different histotypes of cervical carcinoma, one of them originating from two different foci, the other one in the same area (Figures 1 and 2). In both cases, the two tumors were microinvasive, with similar natural history.

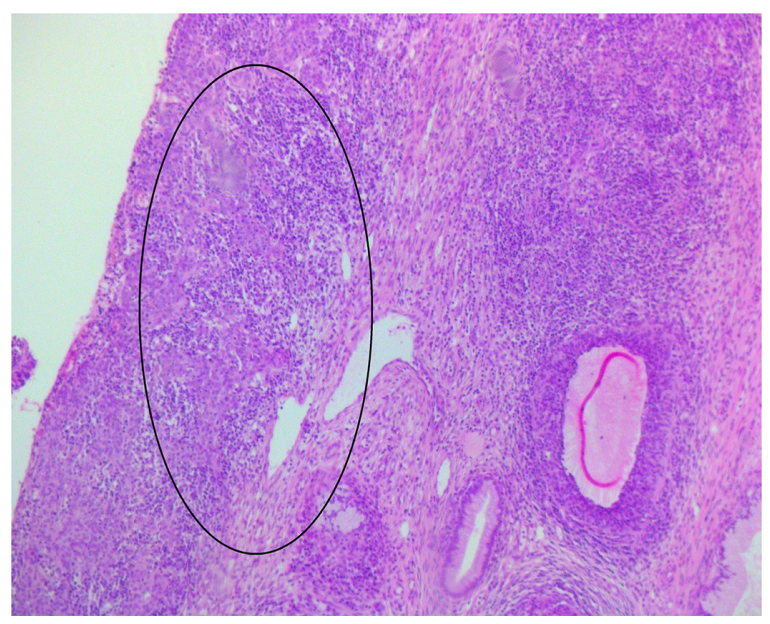

Figure 4 Squamous cell carcinoma (case 2 , original magnification $\times 10$ ). Area inside the oval shows the superficial invasion of cervical stroma.

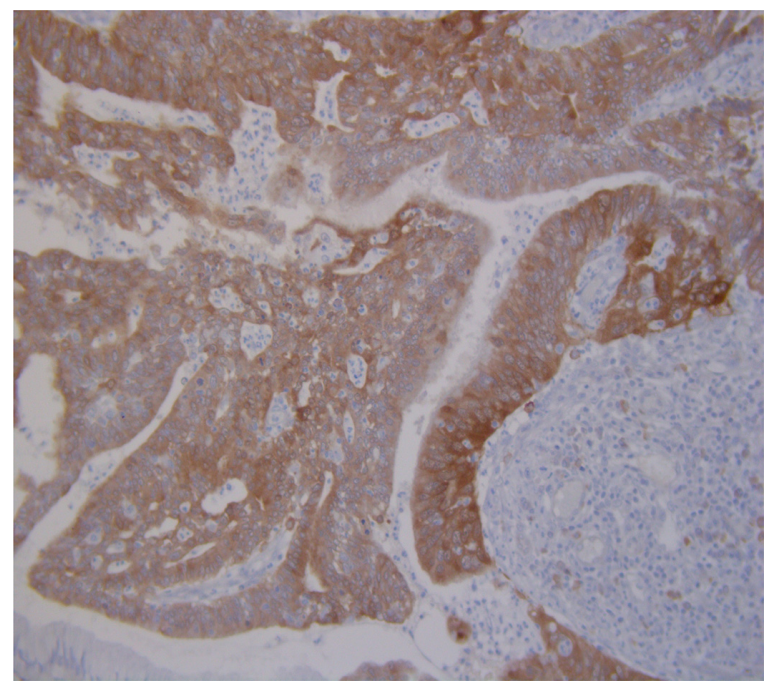

Figure 5 pl6 immunopositive staining in adenocarcinoma cells (case 2, original magnification $\times 10$ ).

A relevant pertinent literature concerns the coexistence of squamous and glandular abnormalities, but no synchronous cases of MISCC and MIAC were reported.

Different degrees of cervical squamous intraepithelial neoplasia and primary adenocarcinoma have been reported. ${ }^{18-20}$ van Aspert-van Erp et al $^{21}$ in 2004, reported 36 cases of severe cervical glandular cell lesions with coexisting squamous cell lesions. Atypical cervical glandular cell lesion, AIS, and CIN were variably coexisting.

In 2014, Pap tests from a series of 260 patients with dual squamous and glandular abnormalities were reported. ${ }^{22}$ Biopsy diagnoses included nine patients with coexistent AIS and CIN. In 1997, Mulvany and Ostor ${ }^{23}$ described a series of 40 cases of MIAC. In 24 cases, CIN 3 and MIAC were coexisting.

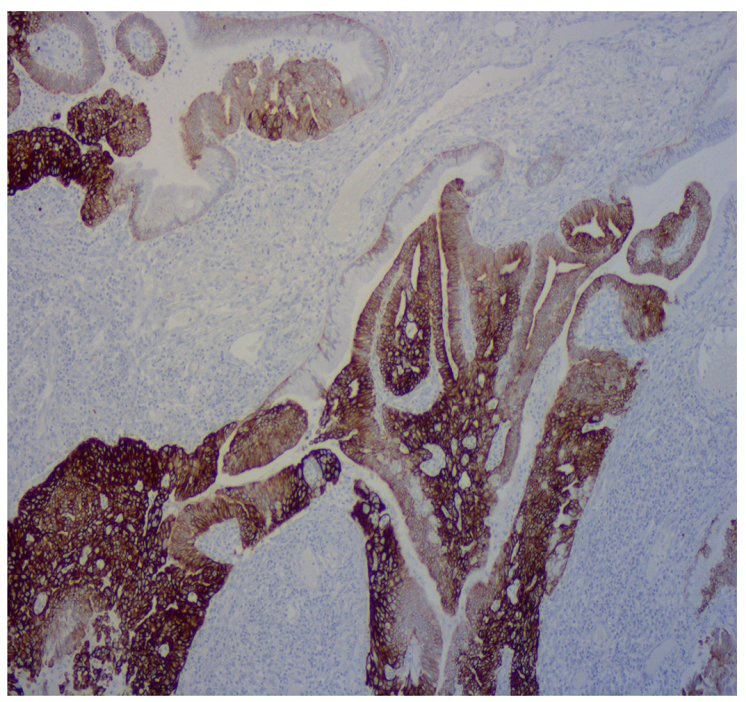

Figure $634 \beta \mathrm{E} / 2$ immunopositive staining in adenocarcinoma and squamous carcinoma cells (case 2 , original magnification $\times 10$ ). 
In 2009, Quint et al ${ }^{24}$ reported 71 cases of cervical adenocarcinomas (31 in situ and 40 invasive) showing that the majority of AIS cases ( $n=24,77.4 \%)$ coexisted with H-SIL. H-SIL was significantly less common in cases of AIS + invasive adenocarcinomas $(n=9,39.1 \%)$ and pure invasive adenocarcinomas $(\mathrm{n}=4,25.0 \%)$.

Very rarely, the concomitant presence of invasive squamous cell carcinoma and invasive adenocarcinoma occurring independently in the cervix has been reported. ${ }^{25,26}$ Goto et a $1^{17}$ in 2005 described a case of a 47 -year-old premenopausal woman with a combination of squamous cell carcinoma and clear cell adenocarcinoma. Based on the diagnosis of stage 1B cervical cancer, a radical hysterectomy with systemic pelvic lymphadenectomy was performed. Parametria and adnexa were free of tumor. She has been followed for over 70 months, with no evidence of recurrent disease.

Interestingly, no patients with coexisting MISCC and MIAC treated conservatively have been reported in the literature and, to our knowledge, the cases presented here are the only available experiences. To date, over 50 types of HPV are known to infect the female genital tract and a subgroup of high-risk HPVs is known to have oncogenic potential. HPV DNA is detected in $>90 \%$ of squamous cell carcinomas of the cervix $;{ }^{27}$ some authors reported a prevalence of HPV DNA in adenocarcinomas in very high percentages, even over $90 \%,{ }^{28,29}$ also suggesting that at least "usual type" adenocarcinomas and squamous cell carcinomas share common HPV etiology. However, recently, a large multicenter study ${ }^{30}$ analyzing 718 cases of cervical adenocarcinomas showed a single HPV genotype in $60 \%$ and $78 \%$ of cases of usual and adenosquamous cervical carcinoma, respectively. In some cases, HPV was found in the adjacent cervix but not in the tumor.

Because of the rarity of coexistence of invasive squamous cell carcinoma and invasive adenocarcinoma of the cervix, there is only one report of HPV status analysis in such double primary carcinoma. ${ }^{17}$ Goto et a ${ }^{17}$ found HPV 18 only in the squamous cell carcinoma and not in the clear cell adenocarcinoma.

In 2014, Kawakami et al ${ }^{31}$ examined eight cases of combined H-SIL with AIS and proposed two developmental pathways of combined H-SIL with AIS. The authors examined the HPV subtypes, the methylation status of the HPV-16 L1 gene, and the immunohistochemical staining pattern of Krt7. With respect to HPV subtype, $75 \%$ of cases showed the same subtype in both H-SIL and AIS, mostly HPV-16 (62.5\%). According to their results, combined H-SIL and AIS lesions seem to arise from the same progenitor cells located at SCJ (common pathway). However, in case of different subtypes of HPV infection, different Krt7 immunohistochemical staining pattern or different status of the HPV-16 L1 gene methylation, Kawakami et al hypothesized that two collision tumors may arise individually from different progenitor cells with different HPV infectious backgrounds (individual pathway). The authors concluded that all their eight cases were treated by hysterectomy because the surgical margins of conization were all positive or very close for AIS, not recommending a conservative approach.

In fact, because of multifocal disease and skip lesions, there may be residual or recurrent glandular neoplasia even in the case of apparently negative surgical margins in cases treated conservatively. ${ }^{32}$ This situation is more dangerous and feared, since follow-up methods are unreliable for endocervical glandular lesions. ${ }^{32}$ Nevertheless, it is now recognized that the histological cell type (squamous or glandular) has no impact on survival for stage I diseases. ${ }^{33}$

In our two cases, a multidisciplinary counseling was offered after definitive histology, and particular attention was called to the limits of a conservative treatment. However, both patients did not consider their reproductive life finished and refused definitive surgery.

\section{Conclusion}

To the best of our knowledge, there are not enough data to classify the choice of conservative treatment for coexisting MISCC and MIAC as safe. At 11 years from the conization, both patients were alive and free of disease, with negative results as far as Pap smear and colposcopy were considered. In women who strongly desire to maintain fertility, affected by stage IA1 squamous cervical cancer coexisting with stage IA1 adenocarcinoma endocervical type, with clear margins and without LVSI, cervical conization may be considered as a definitive treatment, after informed consent by the women. Further cases may be helpful to better define the management of coexisting MISCC and MIAC in women who desire to preserve their fertility.

\section{Disclosure}

The authors report no conflicts of interest in this work.

\section{References}

1. Pecorelli S, Zigliani L, Odicino F. Revised FIGO staging for carcinoma of the cervix. Int J Gynaecol Obstet. 2009;105:107-108.

2. Furtado Y, Almeida G, Lima R, Silva K, Maldonado P. Microinvasive squamous carcinoma (FIGO stage IA1) of the cervix: are there colposcopic criteria for the diagnosis? Am J Obstet Gynecol. 2011; 205:360. 
3. Pettersson BF, Andersson S, Hellman K, Hellstrom AC. Invasive carcinoma of the uterine cervix associated with pregnancy: 90 years of experience. Cancer. 2010;116:2343-2349.

4. Sopracordevole F, Chiossi G, Barbero M, et al. Surgical approach and long-term clinical outcome in women with microinvasive cervical cancer. Anticancer Res. 2014;34:4345-4349.

5. Lee SJ, Kim WY, Lee JW, et al. Conization using electrosurgical conization and cold coagulation for international federation of gynecology and obstetrics stage IA1 squamous cell carcinomas of the uterine cervix. Int J Gynecol Cancer. 2009;19:407-411.

6. Wright JD, NathavithArana R, Lewin SN, et al. Fertility-conserving surgery for young women with stage IA1 cervical cancer: safety and access. Obstet Gynecol. 2010;115:585-590.

7. Takeda N, Suzuki T, Suzuki M, et al. Evaluation of cervical conization as a definitive treatment for microinvasive cervical carcinoma and cervical intraepithelial neoplasia grade 3. Arch Gynecol Obstet. 2012;285: 453-457.

8. Gadducci A, Sartori E, Maggino T, et al. The clinical outcome of patients with stage Ia1 and Ia 2 squamous cell carcinoma of the uterine cervix: a Cooperation Task Force (CTF) study. Eur J Gynaecol Oncol. 2003; 24:513-516.

9. Kodama J, Mizutani Y, Hongo A, Yoshinouchi M, Kudo T, Okuda H. Optimal surgery and diagnostic approach of stage IA2 squamous cell carcinoma of the cervix. Eur J Obstet Gynecol Reprod Biol. 2002;101: 192-195.

10. Rogers LJ, Luesley DM. Stage IA2 cervical carcinoma: how much treatment is enough? Int J Gynecol Cancer. 2009;19:1620-1624.

11. Smith HO, Padilla LA. Adenocarcinoma in situ of the cervix: sensitivity of detection by cervical smear: will cytologic screening for adenocarcinoma in situ reduce incidence rates for adenocarcinoma. Cancer. 2002; 96:319-322.

12. Ostor AG. Early invasive adenocarcinoma of the uterine cervix. Int $J$ Gynecol Pathol. 2000;19:29-38.

13. Hou J, Goldberg GL, Qualls CR, Kuo DY, Forman A, Smith HO. Risk factors for poor prognosis in microinvasive adenocarcinoma of the uterine cervix (IA1 and IA2): a pooled analysis. Gynecol Oncol. 2011;121: $135-142$.

14. López J, Ruíz G, Organista-Nava J, Gariglio P, García-Carrancá A. Human papillomavirus infections and cancer stem cells of tumors from the uterine cervix. Open Virol J. 2012;6:232-240.

15. Michael S, Lambert PF, Strati K. The HPV16 oncogenes cause aberrant stem cell mobilization. Virology. 2013;443:218-225.

16. Jordan J, Martin-Hirsch P, Arbyn M, et al. European guidelines for clinical management of abnormal cervical cytology, part 2. Cytopathology. 2009;20:5-16.

17. Goto K, Takeuchi Y, Yakihara A, Kotsuji F. Synchronous invasive squamous cell carcinoma and clear cell adenocarcinoma of the uterine cervix: a different human papillomavirus status. Gynecol Oncol. 2005;97: 976-979.

18. Jaworski RC, Pacey NF, Greenberg ML, Osborn RA. The histologic diagnosis of adenocarcinoma in situ and related lesions of the cervix uteri. Adenocarcinoma in situ. Cancer. 1988;61:1171-1181.
19. Keyhani-Rofagha S, Brewer J, Prokorym P. Comparative cytologic findings of in situ and invasive adenocarcinoma of the uterine cervix. Diagn Cytopathol. 1995;12:120-125.

20. Ayer B, Pacey F, Greenberg M, Bousfield L. The cytologic diagnosis of adenocarcinoma in situ of the cervix uteri and related lesions. I. Adenocarcinoma in situ. Acta Cytol. 1987;31:397-411.

21. van Aspert-van Erp AJ, Smedts FM, Vooijs GP. Severe cervical glandular cell lesions with coexisting squamous cell lesions. Cancer. 2004;102:218-227.

22. Khor LY, Abdul-Karim FW, Bruening AE, Weber Noffsinger DK, Booth CN. Papanicolaou tests with coexisting squamous and glandular abnormalities. Cancer Cytopathol. 2014;122:620-626.

23. Mulvany N, Ostor A. Microinvasive adenocarcinoma of the cervix: a cytohistopathologic study of 40 cases. Diagn Cytopathol. 1997;16: 430-436.

24. Quint KD, de Koning MN, Geraets DT, Quint WG, Pirog EC. Comprehensive analysis of Human papillomavirus and Chlamydia trachomatis in in-situ and invasive cervical adenocarcinoma. Gynecol Oncol. 2009; 114(3):390-394.

25. Maier RC, Norris HJ. Coexistence of cervical intraepithelial neoplasia with primary adenocarcinoma of the endocervix. Obstet Gynecol. 1980;56:361-364

26. Choo YC, Naylor B. Coexistent squamous cell carcinoma and adenocarcinoma of the uterine cervix. Gynecol Oncol. 1984;17:168-174.

27. Bosch FX, Lorincz A, Munoz N, Meijer CJ, Shah KV. The causal relation between human papillomavirus and cervical cancer. J Clin Pathol. 2002;55:244-265

28. Bray F, Carstensen B, Møller H, et al. Incidence trends of adenocarcinoma of the cervix in 13 European countries. Cancer Epidemiol Biomarkers Prev. 2005;14(9):2191-2199.

29. Holl K, Nowakowski AM, Powell N, et al. Human papillomavirus prevalence and type-distribution in cervical glandular neoplasias: results from a European multinational epidemiological study. Int J Cancer. 2015;137:2858-2868.

30. Molijn A, Jenkins D, Chen W, et al. The complex relationship between human papillomavirus and cervical adenocarcinoma. Int J Cancer. Epub 2015 Aug 8.

31. Kawakami F, Semba S, Itami H, et al. Pathogenesis of combined highgrade squamous intraepithelial lesion and adenocarcinoma in situ of the uterine cervix: human papillomavirus genotype and methylation status and immunohistochemical study. Kobe J Med Sci. 2014;60:E66-E73.

32. Sopracordevole F, Rossi D, Di Giuseppe J, et al. Conservative treatment of stage IA1 adenocarcinoma of the uterine cervix during pregnancy: case report and review of the literature. Case Rep Obstet Gynecol. 2014;2014:296253.

33. Shingleton HM, Bell MC, Fremgen A, et al. Is there really a difference in survival of women with squamous cell carcinoma, adenocarcinoma, and adenosquamous cell carcinoma of the cervix? Cancer. 1995;76: 1948-1955.
OncoTargets and Therapy

\section{Publish your work in this journal}

OncoTargets and Therapy is an international, peer-reviewed, open access journal focusing on the pathological basis of all cancers, potential targets for therapy and treatment protocols employed to improve the management of cancer patients. The journal also focuses on the impact of management programs and new therapeutic agents and protocols on

\section{Dovepress}

patient perspectives such as quality of life, adherence and satisfaction The manuscript management system is completely online and includes a very quick and fair peer-review system, which is all easy to use. Visit http://www.dovepress.com/testimonials.php to read real quotes from published authors. 